\title{
Dosing Time-Dependent Changes in the Anti-tumor Effect of xCT Inhibitor Erastin in Human Breast Cancer Xenograft Mice
}

\author{
Shoya Shiromizu, ${ }^{a}$ Tomoaki Yamauchi, ${ }^{a}$ Naoki Kusunose,${ }^{a}$ Naoya Matsunaga,,${ }^{a, b}$ \\ Satoru Koyanagi, ${ }^{*, a, b}$ and Shigehiro Ohdo*,a \\ ${ }^{a}$ Department of Pharmaceutics, Faculty of Pharmaceutical Sciences, Kyushu University; 3-1-1 Maidashi, Higashi- \\ ku, Fukuoka 812-8582, Japan: and ${ }^{b}$ Department of Glocal Healthcare, Faculty of Pharmaceutical Sciences, Kyushu \\ University; 3-1-1 Maidashi, Higashi-ku, Fukuoka 812-8582, Japan. \\ Received July 2, 2019; accepted August 19, 2019
}

\begin{abstract}
Growth of cancer cells is more highly dependent on various types of amino acids than that of normal cells, and thus prevention of amino acid requirement has been recognized as strategies for cancer therapies. In this study, we found that deprivation of cysteine (Cys) in culturing media prevented the growth of various types of human cancer cell lines. Cys is easily converted to cystine (Cys-Cys) in media and uptaken into cells by cystine/glutamate transporter (xCT). The incorporated Cys-Cys is decomposed into Cys, and used for synthesis of glutathione that suppresses reactive oxygen species-induced cell damage. Therefore, we examined whether a selective $\mathrm{xCT}$ inhibitor erastin prevented the growth of human cancer cell lines. As a result, erastin significantly prevented the proliferation of various types of human cancer cells. Among them, MDA-MB-231 breast cancer cells were identified as the most erastin-sensitive cells. To investigate the ability of erastin to prevent growth of tumor in mice, MDA-MB-231 breast cancer cells were implanted into BALB/c nude female mice kept under standardized light/dark cycle conditions. The growth of tumor implanted in mice was significantly suppressed by administration of erastin during the light phase, whereas its administration during the dark phase failed to suppress the tumor growth. The dosing time-dependency of erastin-induced cystine/ cysteine deprivation was closely related to that of its anti-tumor effects. Our present findings suggest that the anti-tumor efficacy of erastin in tumor-bearing mice is improved by optimizing the dosing schedule.
\end{abstract}

Key words erastin; cystine/glutamate transporter (xCT); cysteine; glutathione; chronopharmacotherapy

\section{INTRODUCTION}

One approach for improving the efficacy of pharmacotherapy is to administer the drugs at the time of day when they are most effective or best tolerated. This strategy is named as chronopharmacotherapy that is based on the internal circadian clock system. Circadian (approximately 24-h) rhythms in various physiological functions are generated by the molecular clock system consisting of clock genes. ${ }^{1-3)}$ The molecular oscillator not only regulates the 24-h rhythms in physiological function but also affects the expression of drugtargeted molecules. ${ }^{4-6)}$ Consequently, administration of drugs at the appropriate times of day can improve the outcome of pharmacotherapy by maximizing the potency and minimizing the toxicity of drugs. ${ }^{7,8)}$ On the other hand, administration of drugs at an inappropriate time of day induces severe side effects. ${ }^{910)}$ Despite the importance of dosing time-dependent change in the drug potency, many drugs are still administered without regard to the times of day.

Many types of cancer cells increase demand for specific amino acids, which is dependent on either exogenous supply or upregulated de novo synthesis. ${ }^{11)}$ Consequently, such amino acid addictions of cancer cells have been recognized as potential target for development of therapies. It has been reported that cysteine (Cys) is an essential amino acid for cancer cell proliferation. ${ }^{12,13)}$ Cys is converted to cystine (Cys-Cys) in cell culture media and uptaken into cells by cystine/glutamate transporter $(\mathrm{xCT})$. The incorporated Cys-Cys is decomposed into $\mathrm{Cys}$ and directly incorporated into glutathione which makes it possible for cells to resist to the surrounding oxidative stress and survive. ${ }^{14)}$ Therefore, strategy to prevent Cys uptake by blocking $\mathrm{xCT}$ is likely to be effective for treatment of cancers.

In this study, we investigated the possibility that anti-tumor activity of a selective $\mathrm{xCT}$ inhibitor erastin in tumor-bearing mice could be improved by changing the administration time. We searched for erastin-sensitive human cancer cell lines and selected MDA-MB-231 breast cancer cells. The cells were implanted into BALB/c nude female mice and influence of dosing time on the anti-tumor activity of erastin was investigated in MDA-MB-231 xenograft mice.

\section{MATERIALS AND METHODS}

Cell Culture and Treatments The human cancer cell lines (HeLa, SK-OV-3, HepG2, A549, H1975, MCF7, T-47D, SK-BR-3 and MDA-MB-231) were maintained in Dulbecco's modified Eagle's medium (DMEM, Sigma-Aldrich, St. Louis, MO, U.S.A.) or RPMI 1640 (Thermo Fisher Scientific, Waltham, MA, U.S.A.) supplemented with 2 or $10 \%$ fetal bovine serum (FBS, Thermo Fisher Scientific) and 0.5\% penicillin-streptomycin (Thermo Fisher Scientific) at $37^{\circ} \mathrm{C}$ in a humidified $5 \% \mathrm{CO}_{2}$ atmosphere. Custom-made amino acid-deficient medium was purchased from Funakoshi Co., Ltd. (Tokyo, Japan). Cells were seeded on 96-well plates and treated with erastin (Sigma-Aldrich) at the indicated concentrations. Cellular growth ability was assessed using a CellTiter-Glo Luminescent Cell Viability Assay (Promega, Madison, WI, U.S.A.). 

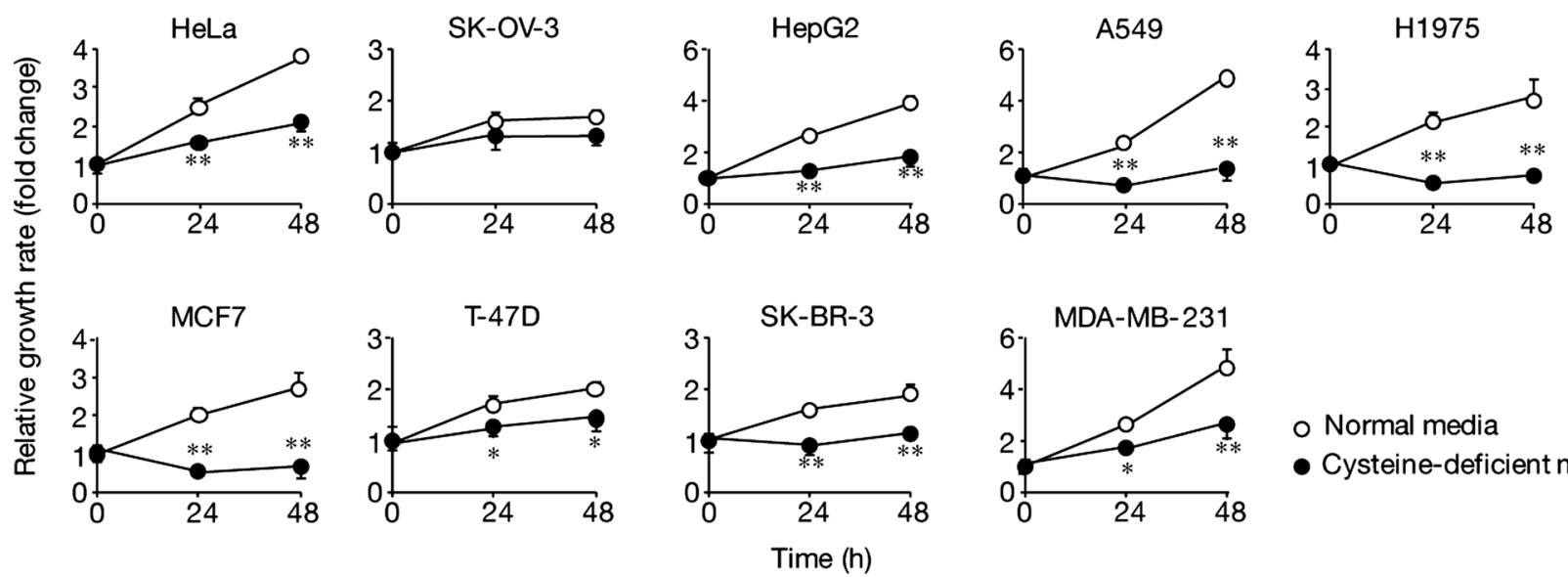

o Normal media

- Cysteine-deficient media

Fig. 1. Suppression of the Growth of Human Cancer Cell Lines in Cysteine-Deficient Media

The human cancer cells (HeLa, SK-OV-3, HepG2, A549, H1975, MCF7, T-47D, SK-BR-3 and MDA-MB-231) were incubated in normal or Cys-deficient media. Cell viability at $0 \mathrm{~h}$ was set to 1.0 . Values represent the mean with standard deviation (S.D.) $(n=4) .{ }^{* *} p<0.01,{ }^{*} p<0.05$ significantly different from normal media group at corresponding time points (Student's $t$-test).

Animals and Treatments Female BALB/c nu/nu mice (Charles River Laboratories, Wilmington, MA, U.S.A.) were housed under a 12-h light/dark cycle at room temperature of $24 \pm 1{ }^{\circ} \mathrm{C}$ and a humidity of $60 \pm 10 \%$ with food and water ad libitum. Under the light/dark cycle, 7:00 was designated as lights on and 19:00 as lights off. During the dark period, a dim red light was used to aid treatment of the mice. A $30-\mu \mathrm{L}$ volume containing $5 \times 10^{6}$ viable MDA-MB-231 tumor cells were subcutaneously inoculated into the back of mice and the diameters of tumors were measured with a caliper. Tumor volumes were calculated using the formula of an ellipsoid [tumor volume $\left(\mathrm{mm}^{3}\right)=4 \pi(X Y Z) / 3$, where $2 X, 2 Y$, and $2 Z$ are the three perpendicular diameters of tumor]. All animal experiments were performed in accordance with the guidelines established by the Animal Care and Use Committee of Kyushu University.

Determination of Intracellular Cys Contents To determine intracellular contents of Cys, cells were washed with phosphate buffered saline, and then homogenized with $550 \mu \mathrm{L}$ methanol containing internal standard $\left(91 \mathrm{ng} / \mathrm{mL}\right.$ L- $\left[1-{ }^{13} \mathrm{C}\right]$ valine). The homogenates were centrifuged at $12000 \times \boldsymbol{g}$ for $5 \mathrm{~min}$ at $4^{\circ} \mathrm{C}$, and the supernatants were filtered using $0.22 \mu \mathrm{m}$ hydrophilic polyvinylidene difluoride membrane (Merk Millipore, Burlington, MA, U.S.A.). After filtration, samples were dried out, and dissolved into $50 \mu \mathrm{L}$ mobile phase. The liquid chromatography tandem mass spectrometer system was ACQUITY (Waters, Milford, MA, U.S.A.). Liquid chromatography was performed using Intrada amino acid column ( $3 \mu \mathrm{m}, 50 \times 3 \mathrm{~mm}$; Imtakt Co., Kyoto, Japan) under gradient elution program. The mobile phases consisted of solvent A (acetonitrile-tetrahydrofuran- $25 \mathrm{mM}$ ammonium formateformic acid $=9: 75: 16: 0.3, \mathrm{v} / \mathrm{v} / \mathrm{v} / \mathrm{v}$ ) and solvent $\mathrm{B}$ (acetonitrile $-100 \mathrm{mM}$ ammonium formate $=1: 4, \mathrm{v} / \mathrm{v}$ ). The following gradient program was applied: $0 \%$ solvent $\mathrm{B}$ at $0 \mathrm{~min}, 0 \%$ solvent $\mathrm{B}$ at $2.5 \mathrm{~min}, 17 \%$ solvent $\mathrm{B}$ at $6.5 \mathrm{~min}, 100 \%$ solvent $\mathrm{B}$ at $7 \mathrm{~min}, 100 \%$ solvent $\mathrm{B}$ at $11 \mathrm{~min}$, and $0 \%$ solvent $\mathrm{B}$ at $12 \mathrm{~min}$ with a flow rate of $0.6 \mathrm{~mL} / \mathrm{min}$. The total run time was $15 \mathrm{~min}$. The mass spectrometer was operated in the multiple reaction monitoring (MRM) mode using positive electrospray ionization. The MRM was set at 122-76.1 and 119-72 mass to charge ratio $(\mathrm{m} / \mathrm{z})$ for Cys and $\mathrm{L}-\left[1-{ }^{13} \mathrm{C}\right]$ valine, respectively. The amount of Cys was normalized by intracellular protein concentrations.

Measurement of Glutathione Contents in Tumors To determine the intratumoral contents of glutathione, cells were homogenized with $500 \mu \mathrm{L}$ methanol. Homogenates were centrifuged at $12000 \times \boldsymbol{g}$ at $4^{\circ} \mathrm{C}$ for $3 \mathrm{~min}$, and supernatants were collected as samples. Cell homogenates were dried at room temperature under reduced pressure, and the residue was dissolved in $100 \mu \mathrm{L}$ of purified water containing of $1.97 \mu \mathrm{mol}$ 5-sulfosalicylic acid. Total glutathione contents were determined by using the GSSG/GSH Quantification kit (Dojindo, Kumamoto, Japan).

Statistical Analysis The Student's $t$-test or Mann-Whitney $U$-test was used for two independent groups. A 5\% or less probability was considered to be significant.

\section{RESULTS}

Influence of Cys Deprivation on the Growth of Human Cancer Cells To investigate the role of Cys in the growth of cancer cells, a variety of human cancer cells (HeLa, SK-OV-3, HepG2, A549, H1975, MCF7, T-47D, SK-BR-3 and MDA-MB-231) were cultured in Cys-deficient media. Except for SK-OV-3, proliferation of most type of cancer cells was prevented by culturing in Cys-deficient media (Fig. 1). The growth of these tumor cells appeared to be dependent on extracellular supply of Cys.

Next, we investigated whether proliferation of human cancer cells were also prevented by treating with a selective $\mathrm{xCT}$ inhibitor erastin. Similar to the results shown in Fig. 1, the growth of most human cancer cells was suppressed by treatment with erastin (Fig. 2). Among them, the proliferation of MDA-MB-231 breast cancer cells was decreased by $70 \%$ as compared to vehicle treated cells, suggesting that this cell line is more sensitive to erastin. In addition, the treatment with erastin also significantly decreased the contents of Cys and glutathione in MDA-MB-231 cells ( $p<0.01$ for Cys, $p<0.01$ for glutathione, respectively, Fig. 3), indicating that erastininduced inhibition of xCT activity leads to deprivation of intracellular glutathione and growth interruption.

Dosing Time-Dependent Difference in the Ability of Erastin to Suppress the Growth of Tumor Cells Implanted 

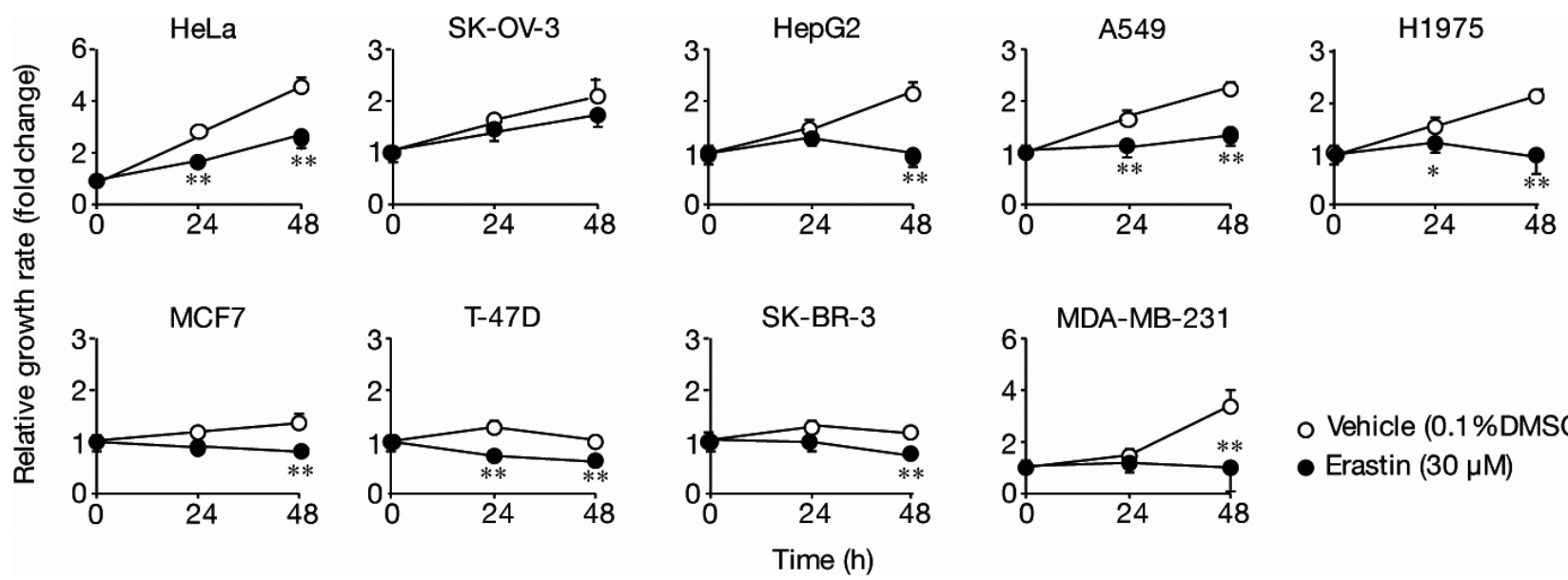

O Vehicle (0.1\%DMSO)
Erastin $(30 \mu \mathrm{M})$

Fig. 2. Inhibitory Effect of Erastin on the Growth of Human Cancer Cell Lines

The human cancer cells (HeLa, SK-OV-3, HepG2, A549, H1975, MCF7, T-47D, SK-BR-3 and MDA-MB-231) were incubated in the media in the presence or absence of $30 \mu \mathrm{M}$ erastin. Cell viability at $0 \mathrm{~h}$ was set to 1.0 . Values represent the mean with S.D. $(n=4)$. ** $p<0.01, * p<0.05$ significantly different from vehicle group at corresponding time points (Student's $t$-test).

A

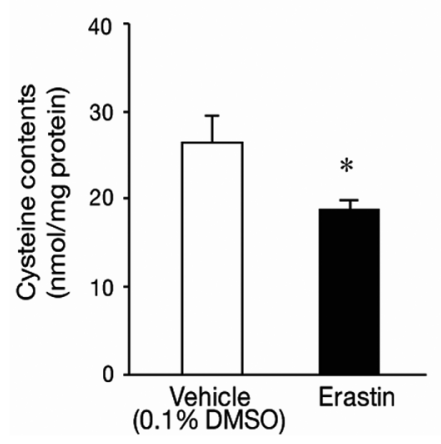

B

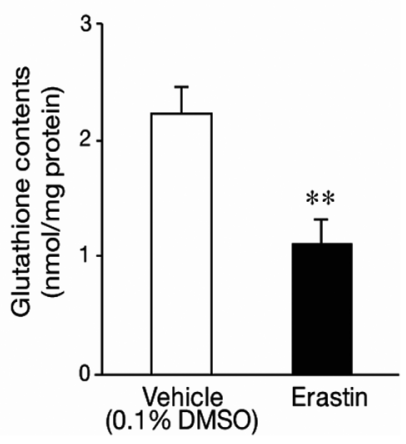

Fig. 3. Influence of Erastin on the Contents of Cysteine and Glutathione in MDA-MB-231 Cells

The amounts of Cys (A) and glutathione (B) in MDA-MB-231 cells were assessed at $24 \mathrm{~h}$ after treatment with erastin. Values represent the mean with S.D. $(n=3-4)$. ${ }^{* *} p<0.01 ;{ }^{*} p<0.05$ significantly different as compared with vehicletreated group $\left(t_{4}=-5.823\right.$ for Cys; $t_{6}=-3.821$ for glutathione, respectively; Student's $t$-test).

in Mice Since proliferation of MDA-MB-231 cells was more severely suppressed by erastin, we further investigated the anti-tumor activity of erastin in MDA-MB-231 tumor-bearing mice. To achieve this, MDA-MB-231 cells were implanted into $\mathrm{BALB} / \mathrm{c} \mathrm{nu} / \mathrm{nu}$ mice, and erastin $(0.5 \mathrm{nmol} / \mathrm{mouse})$ or vehicle (0.1\% dimethyl sulfoxide) were administered subcutaneously at 13:00 or 1:00 every three days (72-h intervals). Mice are nocturnal active. The two dosing time points were selected because of the mid rest phase (13:00) and mid active phase (1:00). The growth of the tumor was significantly suppressed by administration of erastin at 13:00 ( $p<0.05$, Fig. 4A). The mean tumor weight at $21 \mathrm{~d}$ after the initiation of erastin treatment was approximately $50 \%$ smaller than that in vehicletreated mice $(p<0.05$, Fig. $4 \mathrm{~B})$. The treatment with erastin also decreased glutathione contents in tumors $(p<0.05$, Fig. $4 \mathrm{C}$ ), suggesting that suppressive action of this $\mathrm{xCT}$ inhibitor on tumor growth is, at least in part, due to decrease in glutathione contents. On the other hand, the growth of MDAMB-231 cells implanted in mice was not significantly suppressed by treatment with erastin at 1:00 (Fig. 4D). Indeed, the mean tumor weight at $21 \mathrm{~d}$ after the initiation of erastin treatment was comparable to that after vehicle treatment (Fig. 4E).
Since the treatment with erastin during the mid-dark phase had little effect on glutathione contents in tumor cells (Fig. $4 \mathrm{~F}$ ), this may result in failure of the tumor growth inhibition. These results suggest that the ability of erastin to suppress tumor growth varies in a dosing time-dependent manner. Suppressive effect of erastin on the growth of MDA-MB-231 tumors implanted in mice is expected when the inhibitor is administrated during the light phase.

\section{DISCUSSION}

For excessive energy production and rapid proliferation, cancer cells increase demand for glucose and/or specific amino acids. ${ }^{15)}$ A recent clinical trial has shown that Cys is as an essential amino acid for cancer cell proliferation. ${ }^{12,13)}$ Since glutathione alleviated oxidative stress induced by DNA damage, ${ }^{16)}$ it enabled rapid proliferation of cancer cells. Erastin inhibits transport activity of xCT thereby preventing exogenous supply of Cys-Cys to cancer cells. The suppressive effect of erastin on the growth of MDA-MB-231 cells implanted in mice was changed depending on the dosing schedule. These findings raise the possibility that optimizing the dosing schedule could improve the efficacy of drug targeting amino acid metabolism in cancer cells.

Amino acids are essential materials for sustaining cell viability. For example, asparagine and glutamine are supplied to tricarboxylic acid cycle after conversion to oxaloacetic acid and $\alpha$-ketoglutarate, respectively. ${ }^{17)}$ Serine is used for providing one-carbon units to tetrahydrofolate, subsequently contributing to synthesis of nucleic acids with asparagine, glutamine and glycine. ${ }^{18)}$ Cys is directly incorporated into glutathione which makes it possible for cells to resist to the surrounding oxidative stress. ${ }^{14)}$ Since such amino acids are required for proliferation of cancer cells to generate enough energy and to synthesize the biomolecules, deficiency in amino acid supply leads to growth inhibition and/or cell death. The present study demonstrated that the growth of cancer cells was dependent on exogenous supply of Cys. Indeed, prevention of Cys supply to cancer cells by erastin interfered with their proliferation and glutathione synthesis.

On the other hand, daily variations in biological func- 
A

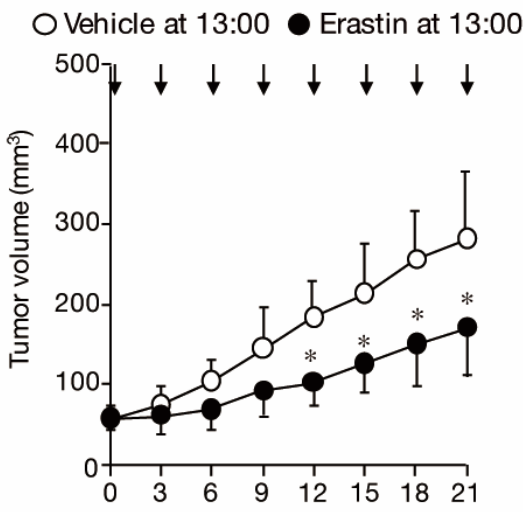

Days after the initiation of erastin treatment

D

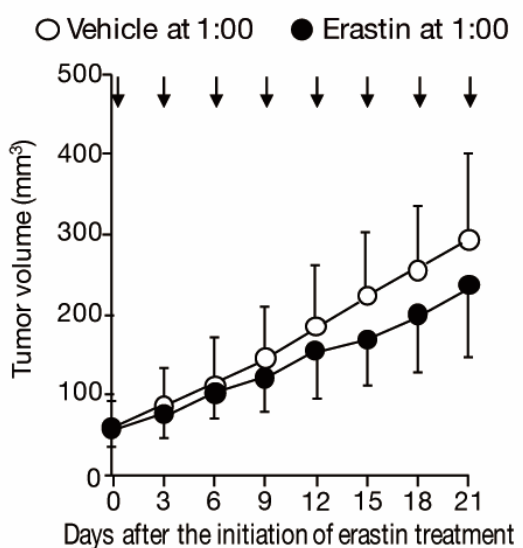

B
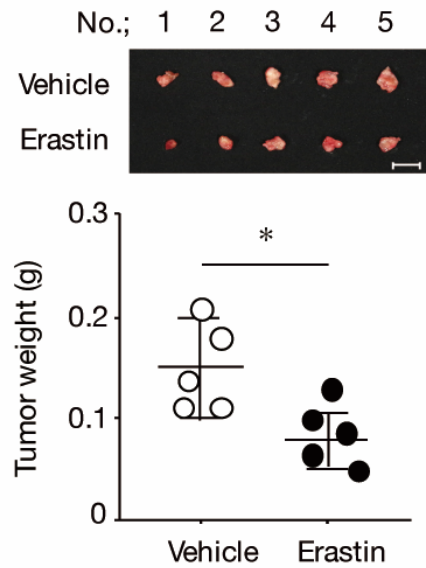

E
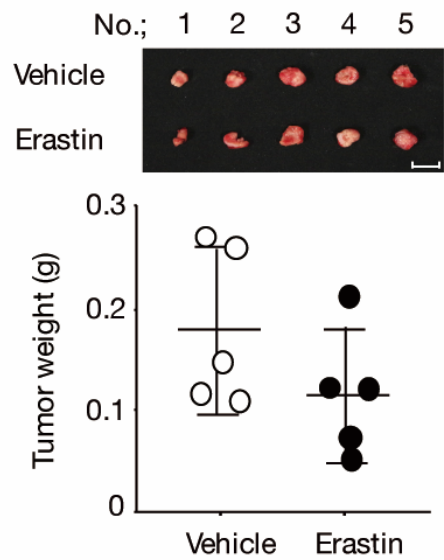

C

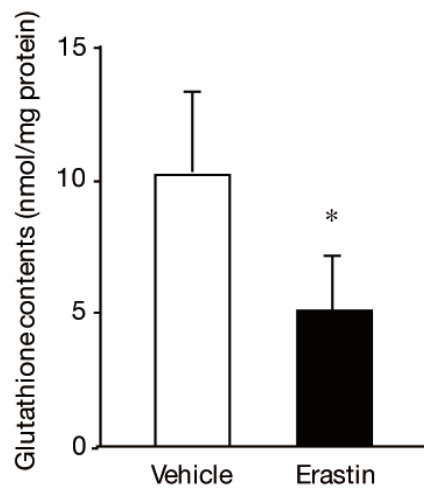

F

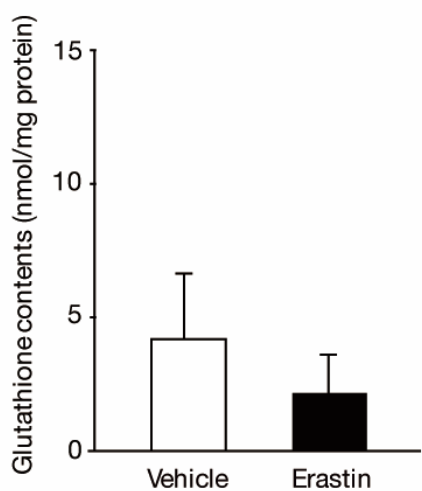

Fig. 4. Dosing Time-Dependent Difference in the Ability of Erastin to Suppress the Growth of MDA-MB-231 Tumor Cells Implanted in Mice

(A) The growth of MDA-MB-231 tumor cells implanted in mice during the repetitive administration of erastin $(0.5 \mathrm{nmol} / \mathrm{mouse})$ or vehicle $(0.1 \%$ DMSO) at $13: 00$. The arrows indicate the administration of erastin or vehicle. Values represent the mean with S.D. $(n=5)$. $* p<0.05$ significantly different from vehicle group at corresponding time points (Student's $t$-test). (B) The upper panel shows the photograph of tumors removed from mice 4 h after the last administration of erastin or vehicle. The unit of scale bar is $10 \mathrm{~mm}$. The lower panel shows the tumor weight. Values represent the mean with S.D. $(n=5)$. ${ }^{*} p<0.05$ significantly different from vehicle group $(z=-2.193$, $p=0.028$; Mann-Whitney $U$-test). (C) Glutathione contents in tumors implanted in mice $4 \mathrm{~h}$ after the last administration of erastin or vehicle. Values represent the mean with S.D. $(n=5) .{ }^{*} p<0.05$ significantly different from vehicle group $(z=-1.984, p=0.047$; Mann-Whitney $U$-test). (D) The growth of MDA-MB-231 tumor cells implanted in mice during the repetitive administration of erastin $(0.5 \mathrm{nmol} / \mathrm{mouse})$ or vehicle $(0.1 \% \mathrm{DMSO})$ at $1: 00$. The arrows indicate the administration of erastin or vehicle. Values represent the mean with S.D. $(n=5)$. (E) The upper panel shows the photograph of tumors removed from mice $4 \mathrm{~h}$ after the last administration of erastin or vehicle. The unit of scale bar is $10 \mathrm{~mm}$. The lower panel shows the tumor weight. Values represent the mean with S.D. ( $n=5$ ). (F) Glutathione contents of tumors removed from mice $4 \mathrm{~h}$ after the last administration of erastin or vehicle. Values represent the mean with S.D. $(n=5)$. (Color figure can be accessed in the online version.)

tions affect the efficacy of drugs. ${ }^{7,8)}$ It has been reported that synthesis and metabolism of amino acids are changed in a circadian time-dependent manner. ${ }^{19)}$ A significant suppressive effect of erastin on the growth of MDA-MB-231 tumors implanted in mice was detected when the drug was administered during the light phase (13:00). During the light phase, DNA synthesis and oxidative stress are enhanced in bone marrow cells and tumors. ${ }^{20,21)}$ Since glutathione contents in the mouse liver have been reported to be increased toward the initiation of the light phase, ${ }^{22}$ tumor cells may also enhance glutathione synthesis during the light phase to alleviate DNA synthesisinduced oxidative stress. Higher glutathione contents in MDAMB-231 cells implanted in mice were detected during the light phase. Glutathione is synthesized via both de novo and salvage synthesis pathways. ${ }^{23)}$ Erastin-induced inhibition of $\mathrm{xCT}$ activity would prevent glutathione synthesis resulting from extracellular supply of Cys-Cys. However, there was no direct evidence of dosing time-dependent difference in the inhibitory effect of erastin on the transport activity of $\mathrm{xCT}$ in MDAMB-231 cells. $\mathrm{xCT}$ is the specific chain of the cystine/glutamate antiporter, and the transport activity varies depending on extracellular Cys-Cys concentrations and intracellular glutamate contents. Although no significant time-dependent variation of xCT expression was detected in MDA-MB-231-bearing mice (Supplementary materials), circadian variation of hepatic glutathione contents in rodents is linked to sulfur amino acid intake. ${ }^{24,25)}$ Since plasma amino acid levels including Cys and Cys-Cys also exhibit circadian variations, ${ }^{26,27)}$ the transport activity of $\mathrm{xCT}$ may also oscillate without changing its expression levels. Such time-dependent variation of $\mathrm{xCT}$ activity seems to affect the inhibitory action of erastin. Further studies are required to elucidate the underlying mechanisms of dosing time-dependent difference in the inhibitory action of erastin on $\mathrm{XCT}$ in tumor cells. 
Accumulated evidence has demonstrated that the effectiveness and toxicity of drugs vary according to their administration time. ${ }^{7,828)}$ Although there is no clinical use of xCTtargeting drug to treat patients with cancers, the present study suggested the importance of optimization of dosing schedule of $\mathrm{xCT}$ inhibitor to suppress tumor growth. Identification of markers for selecting the most appropriate times of day for administration of $\mathrm{xCT}$ inhibitor would help us to achieve rational chronopharmacotherapy targeting amino acid metabolism in cancer cells.

Acknowledgments We appreciate the technical assistance from The Research Support Center, Research Center for Human Disease Modeling, Kyushu University Graduate School of Medical Sciences. This work was supported in part by KAKENHI Grants-in-Aid for Scientific Research $17 \mathrm{H} 06262$ (to S.O.) and 18H04019 (to S.K.) from the Japan Society for the Promotion of Science (JSPS) and Platform Project for Supporting Drug Discovery and Life Science Research [Basis for Supporting Innovative Drug Discovery and Life Science Research (BINDS)] from AMED (Grant Number JP18am0101091).

Conflict of Interest The authors declare no conflict of interest.

Supplementary Materials The online version of this article contains supplementary materials.

\section{REFERENCES}

1) Gekakis N, Staknis D, Nguyen HB, Davis FC, Wilsbacher LD, King DP, Takahashi JS, Weitz CJ. Role of the CLOCK protein in the mammalian circadian mechanism. Science, 280, 1564-1569 (1998).

2) Kume K, Zylka MJ, Sriram S, Shearman LP, Weaver DR, Jin X, Maywood ES, Hastings MH, Reppert SM. mCRY1 and mCRY2 are essential components of the negative limb of the circadian clock feedback loop. Cell, 98, 193-205 (1999).

3) Kramer A, Yang FC, Snodgrass P, Li X, Scammell TE, Davis FC, Weitz CJ. Regulation of daily locomotor activity and sleep by hypothalamic EGF receptor signaling. Science, 294, 2511-2515 (2001).

4) Koyanagi S, Kuramoto Y, Nakagawa H, Aramaki H, Ohdo S, Soeda $\mathrm{S}$, Shimeno H. A molecular mechanism regulating circadian expression of vascular endothelial growth factor in tumor cells. Cancer Res., 63, 7277-7283 (2003).

5) Kanemitsu T, Tsurudome Y, Kusunose N, Oda M, Matsunaga N, Koyanagi S, Ohdo S. Periodic variation in bile acids controls circadian changes in uric acid via regulation of xanthine oxidase by the orphan nuclear receptor PPAR $\alpha$. J. Biol. Chem., 292, 21397-21406 (2017).

6) Horiguchi M, Koyanagi S, Hamdan AM, Kakimoto K, Matsunaga N, Yamashita C, Ohdo S. Rhythmic control of the ARF-MDM2 pathway by ATF4 underlies circadian accumulation of p53 in malignant cells. Cancer Res., 73, 2639-2649 (2013).

7) Lemmer B. Chronopharmacology and controlled drug release. Expert Opin. Drug Deliv., 2, 667-681 (2005).

8) Ohdo S. Chronopharmaceutics: pharmaceutics focused on biological rhythm. Biol. Pharm. Bull., 33, 159-167 (2010).

9) Oda M, Koyanagi S, Tsurudome Y, Kanemitsu T, Matsunaga N, Ohdo S. Renal circadian clock regulates the dosing-time dependency of cisplatin-induced nephrotoxicity in mice. Mol. Pharmacol., 85, 715-722 (2014)

10) Ohdo S, Koyanagi S, Suyama H, Higuchi S, Aramaki H. Changing the dosing schedule minimizes the disruptive effects of interferon on clock function. Nat. Med., 7, 356-360 (2001).

11) Hensley CT, Wasti AT, DeBerardinis RJ. Glutamine and cancer cell biology, physiology, and clinical opportunities. J. Clin. Invest., 123, 3678-3684 (2013).

12) Doxsee DW, Gout PW, Kurita T, Lo M, Buckley AR, Wang Y, Xue H, Karp CM, Cutz JC, Cunha GR, Wang YZ. Sulfasalazine-induced cystine starvation: potential use for prostate cancer therapy. Prostate, 67, 162-171 (2007).

13) Shitara K, Doi T, Nagano O, Imamura CK, Ozeki T, Ishii Y, Tsuchihashi K, Takahashi S, Nakajima TE, Hironaka S, Fukutani M, Hasegawa H, Nomura S, Sato A, Einaga Y, Kuwata T, Saya H, Ohtsu A. Dose-escalation study for the targeting of CD44v+ cancer stem cells by sulfasalazine in patients with advanced gastric cancer. Gastric Cancer, 20, 341-349 (2017).

14) Wu G, Fang YZ, Yang S, Lupton JR, Turner ND. Glutathione metabolism and its implications for health. J. Nutr., 134, 489-492 (2004).

15) Vander Heiden MG, Cantley LC, Thompson CB. Understanding the Warburg effect: the metabolic requirements of cell proliferation. Science, 324, 1029-1033 (2009).

16) Gorrini C, Harris IS, Mak TW. Modulation of oxidative stress as an anticancer strategy. Nat. Rev. Drug Discov., 12, 931-947 (2013).

17) Cooper AJL, Shurubor YI, Dorai T, Pinto JT, Isakova EP, Deryabina YI, Denton TT, Krasnikov BF. $\omega$-Amidase: An underappreciated, but important enzyme in l-glutamine and l-asparagine metabolism; Relevance to sulfur and nitrogen metabolism, tumor biology and hyperammonemic diseases. Amino Acids, 48, 1-20 (2016).

18) Kalhan SC, Hanson RW. Resurgence of serine: An often neglected but indispensable amino acid. J. Biol. Chem., 287, 19786-19791 (2012).

19) Huang A, Bao B, Gaskins HR, Liu H, Zhang X, Lu L, Gao S, Shi Y, Zhang M, Shan Y, Feng J, Yao G. Circadian clock gene expression regulates cancer cell growth through glutaminase. Acta Biochim. Biophys. Sin., 46, 409-414 (2014).

20) Smaaland R, Svardal AM, Lote K, Ueland M, Laerum OD. Glutathione content in human bone marrow and circadian stage relation to DNA synthesis. J. Natl. Cancer Inst., 83, 1092-1098 (1991).

21) Zhao N, Yang K, Yang G, Chen D, Tang H, Zhao D, Zhao C. Aberrant expression of clock gene periodl and its correlations with the growth, proliferation and metastasis of buccal squamous cell carcinoma. PLOS ONE, 8, e55894 (2013).

22) Li XM, Metzger G, Filipski E, Boughattas N, Lemaigre G, Hecquet B, Filipski J, Levi F. Pharmacologic modulation of reduced glutathione circadian rhythms with buthionine sulfoximine: relationship with cisplatin toxicity in mice. Toxicol. Appl. Pharmacol., 143, 281-290 (1997)

23) Lushchak VI. Glutathione homeostasis and functions: potential targets for medical interventions. J. Amino Acids, 2012, 736837 (2012).

24) Isaacs J, Binkley F. Glutathione dependent control of protein disulfide-sulfhydryl content by subcellular fractions of hepatic tissue. Biochim. Biophys. Acta, 497, 192-204 (1977).

25) Jaeschke H, Wendel A. Diurnal fluctuation and pharmacological alteration of mouse organ glutathione content. Biochem. Pharmacol., 34, 1029-1033 (1985)

26) Blanco RA, Ziegler TR, Carlson BA, Cheng PY, Park Y, Cotsonis GA, Accardi CJ, Jones DP. Diurnal variation in glutathione and cysteine redox states in human plasma. Am. J. Clin. Nutr., 86, 1016-1023 (2007).

27) Feigin RD, Dangerfield HG, Beisel WR. Circadian periodicity of blood amino acids in normal and adrenalectomized mice. Nature, 221, 94-95 (1969).

28) Kapse S, Ando H, Fujiwara Y, Suzuki C, Ushijima K, Kitamura H, Hosohata K, Kotani K, Shimba S, Fujimura A. Effect of a dosingtime on quetiapine-induced acute hyperglycemia in mice. J. Pharmacol. Sci., 133, 139-145 (2017). 